\title{
Migración y redes sociales: discursos sobre la ayuda social hacia los migrantes venezolanos en contexto de pandemia por los usuarios peruanos en Twitter \\ Migration and Social Networks: Discourses on Social Assistance for Venezuelan Migrants in the Context of the Pandemic by Peruvian Twitter Users
}

Estudiante de pregrado de Lingüística de la Universidad Nacional Mayor de San Marcos (UNMSM). Pertenece al grupo de investigación Lenguas y Filosofías del Perú de la Facultad de Letras y Ciencias Humanas de la UNMSM, donde se desempeña como colaborador y tesista. Actualmente, cursa el décimo ciclo de la carrera y trabaja en su tesis de licenciatura sobre los repertorios interpretativos del comerciante informal en los diarios digitales peruanos durante el aislamiento social obligatorio del año 2020 (marzo-julio). Sus principales campos de interés son el análisis crítico del discurso, la sociolingüística, la etnolingüística y las redes sociales.

MIRELLA ALEXANDRA ROBLES MUÑOZ

Estudiante de Linguística de la Universidad Nacional Mayor de San Marcos. Se interesa por la sociolingüística, la lexicografía y el análisis del discurso. Ha colaborado en publicaciones para las revistas Lengua y Sociedad y Tierra Nuestra. Ha participado como ponente en Expoletras IX2019, en el VII Encuentro de Jóvenes Investigadores en Humanidades 2020 y en el XXII Diálogo de Estudiantes de Lingüística 2021. 



\section{Migración y redes sociales: discursos sobre la ayuda social hacia los migrantes venezolanos en contexto de pandemia por los usuarios peruanos en Twitter ${ }^{1}$ \\ Migration and Social Networks: Discourses on Social Assistance for Venezuelan Migrants in the Context of the Pandemic by Peruvian Twitter Users}

Edgar Mauro Yalta Gonzales y Mirella Alexandra Robles Muñoz

Universidad Nacional Mayor de San Marcos, Perú edgar.yalta@unmsm.edu.pe (https://orcid.org/o0oo-0001-8444-6779)

mirella.robles@unmsm.edu.pe (https://orcid.org/o0oo-0002-9812-1606)

Recibido: 19-05-2021 / Aceptado: 16-09-2021

https://doi.org/10.18800/conexion.202102.004

\section{PALABRAS CLAVE / KEYWORDS}

Discurso, migración, redes sociales, Venezuela, COVID-19 / discourse, migration, social networks, Venezuela, COVID-19

\section{RESUMEN}

La pandemia por la COVID-19 ha desnudado y agudizado los problemas económicos, sanitarios, políticos y sociales que existen en el Perú. Entre ellos, la migración venezolana y su condición de vulnerabilidad durante la pandemia no ha sido la excepción. Por esta razón, se generó un debate, tanto entre la población como en los medios, respecto a los migrantes venezolanos y si estos también deberían recibir apoyo social por parte del Estado. En el presente artículo, planteamos como objetivos analizar los discursos que generaron en sus tuits los usuarios peruanos de la red social Twitter respecto al debate sobre la ayuda social hacia los migrantes venezolanos, e identificar las estrategias y recursos que utilizaron los usuarios para elaborar estos discursos. Para ello, seleccionamos una encuesta en línea propuesta por la cuenta en Twitter del canal de noticias ATV+ Noticias y, para elaborar el corpus, recogimos los tuits más representativos de la sección de comentarios. Los resultados indican que los usuarios peruanos evidencian una marcada posición polarizada con una mayor tendencia a comentar negativamente sobre los migrantes.

\section{ABSTRACT}

The COVID-19 pandemic has exposed and exacerbated the economic, health, political and social problems that exist

${ }^{1}$ Esta investigación fue financiada por la Universidad Nacional Mayor de San Marcos, R. R. N. ${ }^{\circ}$ 05753-R-21 con código de proyecto E21030371. 
in Peru. Among them, the issue of Venezuelan migration and its vulnerability during the pandemic has not been the exception. For this reason, a debate was generated, both among the population and in the media, regarding Venezuelan migrants and whether they should also receive social support from the State. In this article, our objectives are to analyze the discourses generated in their tweets by Peruvian users of the social network Twitter regarding the debate on social support for Venezuelan migrants, and to identify the strategies and resources used by users to elaborate these discourses. To do so, we selected an online survey proposed by the Twitter account of the news channel ATV+ Noticias and collected the most representative tweets from the comments section to elaborate the corpus. The results indicate that Peruvian users show a marked polarized position with a greater tendency to comment negatively on migrants.

\section{Migración y redes sociales: discursos sobre la ayuda social hacia los migrantes venezolanos en contexto de pandemia por los usuarios peruanos en Twitter}

La COVID-19 es una enfermedad infecciosa causada por el virus SARS-CoV-2 que apareció a finales del año 2019 en la ciudad de Wuhan en China y se propagó rápidamente hacia el resto de territorios (Pérez Abreu et al., 2020). El impacto de directamente a todos los países del mundo en sus distintos sectores -economía, salud, política, entre otros-debido a las medidas que se implementaron para reducir la propagación del virus; en consecuencia, se generó una de las recesiones económicas más graves de la historia (Banco Mundial, 2020). En el Perú, el primer caso de la COVID-19 se reportó el 6 de marzo de 2020 y se comunicó a través de un mensaje emitido en una conferencia por el expresidente Martín Vizcarra («Coronavirus en Perú», 2020). Seguidamente, ante el incremento acelerado de casos de contagio por el virus, el Gobierno - a través del Decreto Supremo $\mathrm{N}^{\circ}$ 044-2020-PCM (2020) - limitó la libertad de tránsito y estableció restricciones en los sectores de comercio, producción y transporte. Ante la rigurosidad de las medidas establecidas y la continua extensión del aislamiento social obligatorio durante los meses posteriores, la salud, la economía, la educación, la política y otros sectores sociales en el Perú se vieron desfavorecidos (Barrutia Barreto et al., 2021; Gonzales-Castillo et al., 2020; Huaman Fernández, 2021; Lovón Cueva y Chegne Cortez, 2021). Por ello, el Gobierno proporcionó apoyo -que incluía bonos y subsidios- a las personas y empresas afectadas por las consecuencias del aislamiento social obligatorio, la inmovilización y otras medidas (Gobierno del Perú, 2021); además, promovió - en el sector social y de salud-programas para favorecer a las comunidades y hogares más vulnera- 
bles («Ministra Donayre: Programas sociales se han fortalecido», 2020).

Entre las consecuencias previamente señaladas - para el propósito de esta investigación-, nos enfocamos en el aspecto migratorio; específicamente, en la migración de la población venezolana y su situación de vulnerabilidad durante el contexto de pandemia por la COVID-19 en el Perú. La problemática de los migrantes venezolanos -como población vulnerable durante la pandemia - involucró al Estado peruano en diversos ámbitos: social, económico, de salud, etcétera. Por esta razón, su situación se estableció como un tema polémico y constantemente debatido durante este contexto, no solo por la población, sino también en los medios de comunicación. Los debates en cuestión en medios de comunicación como la prensa, la televisión o las redes sociales se explicitaron a través de discursos que estuvieron influidos por una marcada polarización, que se relacionó con la xenofobia y la inclusión. En el contexto de una virtualidad masiva y globalizada que se impulsó aún más durante la pandemia por la COVID-19, las redes sociales -por ejemplo, Facebook y Twitter- se establecieron como medios productores de debate entre usuarios respecto a las problemáticas relacionadas con el migrante venezolano. Un caso importante es el debate que se produjo durante los últimos meses del año 2020 sobre si el Estado, además de beneficiar a los ciudadanos peruanos, también debía ofrecer apoyo económico y social a los migrantes venezolanos. Debido al impacto de las redes sociales, los diarios y medios televisivos - como moderadores - trasladaron este debate a sus redes sociales a través de la aplicación de encuestas en línea, con la finalidad de conocer la opinión de los usuarios peruanos. De esta manera, estos últimos, por medio de tuits, expresaron sus distintos puntos de vista en discursos - basados en estrategias y recursos lingüísticos específicos- que estuvieron marcadamente polarizados respecto a este debate.

En el presente artículo, nos planteamos como principales objetivos analizar los discursos que generaron en sus tuits los usuarios peruanos de la red social Twitter respecto al debate sobre la ayuda social hacia los migrantes venezolanos en una encuesta en línea durante la pandemia, e identificar las estrategias y recursos discursivos que utilizaron los usuarios para elaborar estos discursos. Además, es importante agregar que la investigación nos permite observar cómo, por medio del discurso y de diversas estrategias y recursos, los usuarios en las redes sociales expresan sus distintos puntos de vista - por ejemplo, el racismo, el rechazo, la xenofobia y la inclusión - ante la desigualdad en el acceso a la ayuda social proporcionada por el Gobierno para las comunidades vulnerables en el Perú. En este sentido, es relevante conocer y analizar estos discursos que se construyen en las redes sociales, ya que, durante la pandemia 
por la COVID-19, los medios virtuales se han fortalecido y se han expandido notablemente; en esta línea, es fundamental que se continúen desarrollando trabajos sobre el discurso en los medios virtuales.

Por último, organizamos el artículo de la siguiente manera: en primer lugar, presentamos el marco conceptual; luego, el marco metodológico, que incluye las redes sociales, la migración venezolana y el análisis crítico del discurso; seguidamente, el análisis de los discursos que se generaron en los tuits de los usuarios peruanos en la consulta en línea; finalmente, las conclusiones y las referencias bibliográficas.

\section{Redes sociales}

Los avances tecnológicos en el ámbito de las comunicaciones han permitido un mayor y mejor acercamiento con una gran masa poblacional. Esto ha posibilitado que se establezcan como portales donde se difunden, se presentan y se discuten temas de distintas índoles y donde los usuarios tienen la seguridad de que sus opiniones son escuchadas, leídas y tomadas en cuenta. Dentro de los medios de comunicación virtual más importantes y recientes, encontramos a las redes sociales. Respecto a estas, Hütt Herrera (2012) apunta que «se han consolidado como herramientas de comunicación dentro de la sociedad, a través de las cuales, tanto individuos como empresas, han logrado información con públicos o grupos específicos» (p. 128); por esta razón, incluso, algunos medios tradicionales - por ejemplo, la televisión o los diarios- encuentran en las redes sociales la posibilidad de llegar a una mayor cantidad de población y hacer partícipes activos a los usuarios de los temas que se exponen. Además, las redes sociales se caracterizan por generar mayor contacto en tiempo real con distintas personas que abarcan el territorio nacional e internacional. El Observatorio Nacional de las Telecomunicaciones y de la SI (2011) agrega que se las considera como espacio democratizador, donde los usuarios son productores, portadores y receptores de información. En esta línea, interpretamos que las redes sociales son un medio de difusión informativo en el que los usuarios generan y consumen discursos expuestos a debate.

En la presente investigación, nos enfocamos en la red social Twitter. Esta red acoge una gran cantidad de usuarios; por ejemplo, según los datos proporcionados por la plataforma DataReportal (2021), Twitter cuenta con un aproximado de 377 millones de usuarios a nivel global y con alrededor de 1.55 millones de usuarios en el Perú. Asimismo, esta red social se caracteriza por mantener su contenido constantemente actualizado; en consecuencia, se generan reacciones inmediatas que invitan al debate. Por otro lado, también es importante destacar los servicios y herramientas divergentes que ofrece Twitter; por ejemplo, conectividad des- 
de diferentes partes - vía web o móvil-, programas de mensajería instantánea (Pérez Matar y Paredes Esponda, 2016), elaboración de encuestas, etiquetas a través de hashtags, menciones a otros usuarios (Gallardo Paúls y Enguix Oliver, 2016), etcétera. Precisamente, estos servicios son los que persuaden a distintas personas, ya que lo perciben como un medio en el que se puede debatir sencillamente en tiempo real en torno a temas actuales que pueden aquejar a una o más nacionalidades, a uno o más territorios y a una persona o a una multitud entera.

Ahora bien, las redes sociales, como Twitter en este caso, al tratarse de espacios abiertos, reproducen comentarios con mayor libertad. En muchas ocasiones, estos comentarios están cargados de sentimientos y mensajes negativos, que pueden responder al conjunto de experiencias que el usuario ha adquirido durante su vida. Estos patrones suelen evidenciarse constantemente en los discursos que generan los usuarios ante un tema o una comunidad que, desde su perspectiva, no es de su agrado. Por ello, estudiar estos discursos permite tener mayor acceso a la manera de pensar y expresarse de un individuo en las redes sociales -especialmente si se trata de valoraciones o juicios-, así como a las herramientas que utilizan para exponer su posición sobre un tópico determinado. Además, es importante tener en cuenta que estos discursos, en ocasiones, se elaboran de manera anónima, lo cual permite al usuario pro- ducir discursos sin filtros; en consecuencia, se generan comentarios polémicos y respuestas inmediatas a estos por parte de otros usuarios.

\section{Migración venezolana}

El desplazamiento de personas de un país a otro -por motivos económicos, políticos, religiosos o culturales- se ha evidenciado a través de la historia en diferentes épocas y de distintas maneras. Este desplazamiento de un territorio nacional a otro con fines residenciales se denomina migración internacional (Gómez Walteros, 2010). Esta migración se manifiesta usualmente en el traslado de población de un país menos desarrollado a uno con mayores posibilidades de subsistencia en un determinado contexto. En los últimos 10 años, el territorio peruano, sobre todo la capital -Lima-, ha experimentado una gran cantidad de oleadas migratorias provenientes de Venezuela. Según los datos proporcionados por la ACNUR (2019), hasta el año 2018 el Perú acogía el tercer mayor número de migrantes venezolanos en Latinoamérica, con un aproximado de 506000 personas. Además, según los informes de la Defensoría del Pueblo (2020), la migración se agudizó debido a la pandemia generada por la COVID-19.

Koechlin et al. (2018) señalan que, a partir del año 2013 y, sobre todo, desde el 2016 en adelante, las migraciones de personas venezolanas al territorio peruano presentan una tendencia ascendente ace- 
lerada. Las razones que sustentan este desequilibrio poblacional en el Perú se originan en la crisis política, económica y social que experimenta Venezuela. Por ello, no se trata de un traslado esporádico u ocasional, sino que el migrante es consciente de la realidad que azota a su país y se traslada, muchas veces de manera involuntaria, al territorio peruano, con la finalidad de asentarse. Según los datos estadísticos que ofrece el Instituto Nacional de Estadística e Informática (2019), el $62.5 \%$ de la población venezolana que emigró al Perú en 2018 dejó algún familiar con quien vivió en su país natal. Además, durante este periodo, los datos estadísticos también reflejan que el $94.7 \%$ tiene intención de quedarse en el Perú, pese a trabajar de manera informal y estar, en su mayoría, sobrecalificados. Aun así, son explotados en los puestos laborales que ejercen.

Esta realidad se mantiene y se agudiza durante el contexto de pandemia por la COVID-19, ya que aún se perciben migrantes venezolanos en trabajos informales y en circunstancias marginales. Si bien es cierto que el Gobierno peruano adoptó políticas de protección al migrante venezolano antes de la pandemia - como la creación del Permiso Temporal de Permanencia (PTP), emitido por Decreto Supremo $\mathrm{N}^{\circ}$ o01-2018-IN y modificado por Decreto Supremo N ${ }^{\circ}$ 007-2018-IN, y la creación del Acta de Permiso de Trabajo Extraordinario provisional hasta culminar el trámite del primero (Superintendencia
Nacional de Migraciones, 2019)-, con la llegada masiva a causa de la COVID-19, la vulnerabilidad del migrante venezolano se acrecentó en el Perú. Por ello, Migraciones adoptó, hasta el término del estado de emergencia que implantó el Gobierno peruano, nuevas medidas dirigidas a sancionar irregularidades migratorias; sin embargo, este hecho vulneró aún más el desarrollo de los migrantes venezolanos en el país, ya que se aplazaron, entre otros, los trámites de regularidad migratoria en calidad de refugiados. Ante ello, la Defensoría del Pueblo (2020) considera que estas medidas limitan las oportunidades de subsistencia de los migrantes en el Perú y pueden provocar prácticas xenofóbicas que vulneren sus derechos.

Las consecuencias ante el impulso migratorio de población venezolana generado por la pandemia causada por la COVID-19 producen una serie de debates acerca de si los migrantes venezolanos deben recibir ayuda en todos los sectores -social, salud, etcétera- en el Perú tomando en cuenta el contexto, en el que la población peruana se siente igual de vulnerable. Es posible que un grupo de personas perciban como amenaza a los migrantes venezolanos, ya que, por ejemplo, en el campo laboral se sienten usualmente desplazados por estos últimos. Uno de los temas específicos, la ayuda social, es motivo de juicios valorativos elaborados por los residentes peruanos. Estas valoraciones se manifiestan en comentarios polarizados. Además, quienes migran no 
solo se desplazan de un país a otro, sino que llevan consigo un bagaje de conocimientos, costumbres y culturas que los identifican como individuos y como parte de una nación. Por lo tanto, llegar al país de destino origina, entre los ciudadanos peruanos, respuestas divergentes, que responden a identidades que pueden converger o repelerse.

\section{Análisis crítico del discurso}

Según Meersohn (2005), el discurso puede ser interpretado «como un evento comunicativo completo en una situación social» (p. 291); además, es importante agregar que el discurso «construye realidad y proporciona una forma común de entender el mundo por los individuos y pragmáticamente, es el lenguaje en uso y sus efectos en los distintos contextos sociales» (Urra et al., 2013, p. 52). El análisis del discurso (AD) busca incluir una relación entre texto y contexto, $\mathrm{y}$, en esta línea, el analista tendrá como finalidad «averiguar los efectos constructivos del discurso a través del estudio sistemático y estructurado de los textos» (Urra et al., 2013, p. 52). Por otro lado, Suurmond (2005) presenta una clasificación en la que organiza el AD en tres subdivisiones, según el tipo de análisis: el análisis del discurso desde el enfoque lingüístico, el análisis conversacional y el análisis del discurso en las ciencias sociales. Dentro de la tercera subdivisión, encontramos la psicología discursiva, el análisis del discurso de Foucault y el análisis crítico del discurso.
Este último es el tipo de análisis en el que se basará nuestra investigación. Respecto al análisis crítico del discurso (ACD), Van Dijk (2015/2016) señala lo siguiente:

Es un tipo de investigación que se centra en el análisis discursivo y estudia, principalmente, la forma en la que el abuso de poder y la desigualdad social se representan, reproducen, legitiman y resisten en el texto y el habla en contextos sociales y políticos (p. 204).

Además, también es importante destacar las propiedades generales del ACD. Van Dijk (2015/2016, p. 205) señala las siguientes: se enfoca en los problemas sociales y las cuestiones políticas, tal como en el caso de esta investigación; además, se caracteriza por ser multidisciplinario; por otro lado, no solo describe la estructura discursiva, sino que también la explica en términos de estructura e interacción social; por último, se enfoca en la manera en que las estructuras discursivas representan las relaciones de dominación en la sociedad.

Por último - como hemos señalado constantemente-, debido a la pandemia por la COVID-19, la virtualidad se ha fortalecido y la interacción de usuarios en las redes sociales se ha establecido como una dinámica muy común en la sociedad actual. Por esta razón, los discursos que se generan en las redes sociales se consideran importantes y se debe tener en cuenta 
que sus mecanismos discursivos ya son objeto de estudio en otras investigaciones (Portillo Fernández, 2016). En el caso de la presente investigación, el ACD nos permite, a través de su aplicación, identificar y analizar las estrategias, los mecanismos y los recursos discursivos - como la selección léxica, la hiperbolización, etcétera- que se encuentran presentes en los discursos generados por los usuarios peruanos en la red social, tanto para expresar el rechazo como la inclusión frente a una comunidad.

\section{Metodología}

La investigación es de carácter cualitativo, explicativo e interpretativo. Seleccionamos este enfoque, ya que nos permite «examinar la forma en que ciertos individuos perciben y experimentan fenómenos que los rodean, profundizando en sus puntos de vista, interpretaciones y significados» (Hernández Sampieri y Mendoza, 2018, p. 390). Asimismo, la investigación se ubica en el campo de estudio de los espacios virtuales; específicamente, en los medios de comunicación virtual. La unidad de análisis son los discursos generados por los usuarios peruanos en sus tuits en la red social Twitter. Estos discursos se presentan en forma de comentarios en una encuesta en línea establecida por la cuenta de Twitter de un medio televisivo. La encuesta en línea que se seleccionó fue la propuesta por la cuenta en Twitter del canal de noticias ATV+ Noticias. La puesta dicotómica y fue publicada el 24 de septiembre de 2020. La pregunta en cuestión fue la siguiente: ¿Cree usted que el Gobierno debe implementar programas de ayuda social para los venezolanos que atraviesan la crisis de la pandemia? La encuesta y los resultados se pueden observar en la Figura 1.

Además, es importante destacar que la encuesta en línea en cuestión fue seleccionada porque presenta un ejemplo de los tipos de debates y discusiones que se generaron entre los usuarios de esta red social durante la pandemia respecto a la ayuda social para los migrantes venezolanos. Por esta razón, los resultados, especialmente los comentarios de los usuarios, son muestra de una perspectiva polarizada que, si bien no es generalizada, es representativa en las redes sociales debido a la pregunta directa que plantea. Este tipo de prácticas - plantear preguntas directas- por parte de los medios permite que los usuarios se sientan «consultados» (Gallardo Paúls y Enguix Oliver, 2016) y, en consecuencia, que tengan una interacción y participación más activa.

Tomando en cuenta la encuesta realizada y la distribución de los resultados, se estudió un conjunto de 20 tuits; a partir de ello, se seleccionaron los 15 tuits más representativos, que permiten dar cuenta de las reacciones polarizadas ante la consulta sobre implementar la ayuda social para los migrantes venezolanos. Por otra parte, los tuits en cuestión datan del 
Figura 1

Resultados de la encuesta realizada por ATV+ Noticias

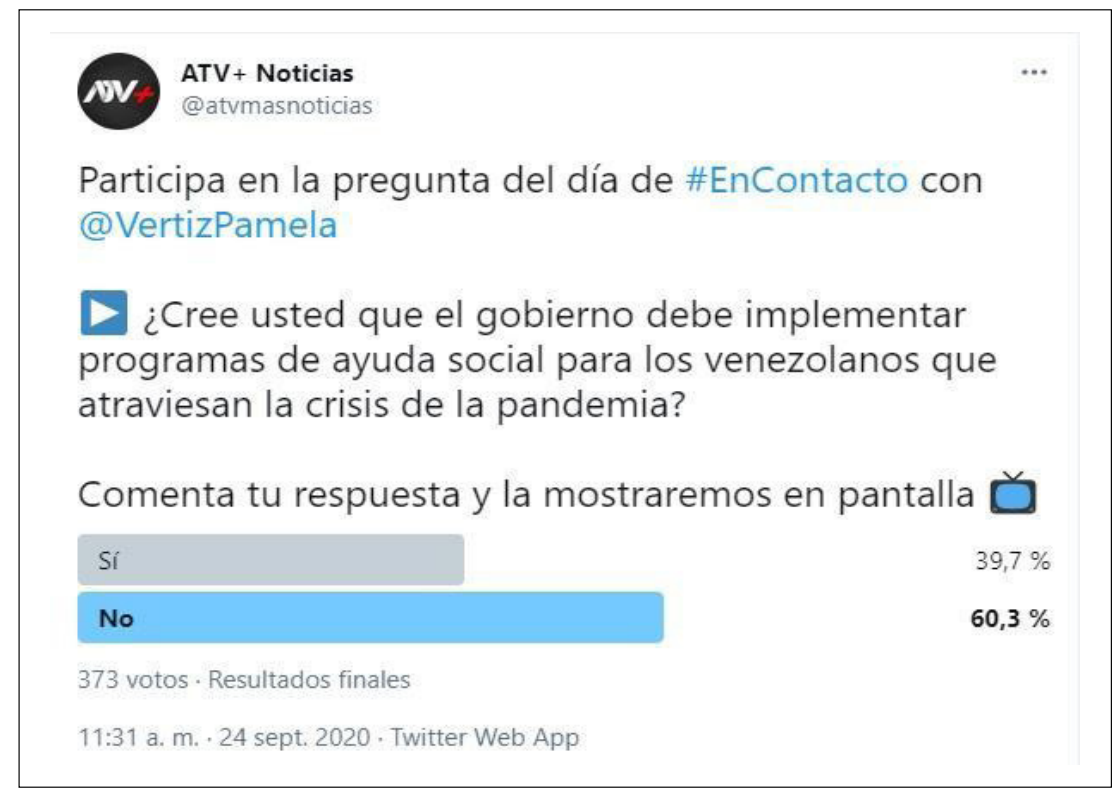

Nota. Captura de pantalla de Participa en la pregunta del día de \#EnContacto con @VertizPamela ¿Cree usted que el gobierno debe implementar programas de ayuda [Tuit], por ATV+ Noticias [@atvmasnoticias], 24 de septiembre de 2020, Twitter (https://twitter.com/atvmasnoticias/status/1309168686259941376).

día en que se publicó la consulta pública, es decir, el 24 de septiembre de 2020. Por último, los tuits analizados se mantienen sin alterar la ortografía y la redacción, ya que explicitan la manera en que los usuarios expresan su punto de vista.

\section{Análisis}

En este apartado, como se mencionó, se analizan los discursos más representativos de los tuits generados como comentarios sobre la encuesta en línea elaborada por la cuenta en Twitter de ATV+ Noticias. Además, se identifican las estrategias y recursos que utilizan los usuarios para elaborar estos discursos. Como ya se señaló anteriormente, los comentarios son marcadamente polarizados, pero hay una mayor inclinación hacia los comentarios negativos respecto a la ayuda social para los migrantes venezolanos. De esta manera, presentamos, en la primera sección, los tuits con respuestas negativas ante la inclusión de los migrantes venezolanos en programas de ayuda social; luego, en la segunda sección, los tuits con respuestas positivas ante la inclusión de migrantes venezolanos en dichos programas. 


\section{«Primero están los peruanos, no tenemos ni para nosotros mismos»}

En esta primera sección, se analizan los tuits con respuestas y comentarios negativos y en contra de la ayuda social para los migrantes venezolanos. El discurso de primero los peruanos y para los peruanos es una constante en los usuarios que respondieron a la consulta en línea en cuestión. Este discurso está basado, principalmente, en el sentido de pertenencia, el nacionalismo y la xenofobia de los usuarios. Observemos, a continuación, el texto del siguiente tuit:

(1) Primero están lo peruanos, hay muchos compatriotas que no tienen ni SIS y a los venezolanos ni bien llegan les dan ese seguro.

La economía peruana ya no puede mantener a esos delincuentes (venezolanos).

La prensa mermelera se presta a esta invasión y no informa la realidad (Rommel, 2020).

En (1), el usuario aplica el criterio de pertenencia al momento de resaltar a los peruanos y compatriotas; de esta manera, excluye a los migrantes venezolanos en el grupo de no peruanos. Esto se relaciona con la estrategia de polarización planteada por Van Dijk (2003), que pretende incluir a un grupo de personas y excluir a otro grupo. Además, también observamos la estrategia en cuestión cuando el usuario señala que los social: primero están los peruanos. Luego, el usuario destaca que incluso ni los mismos peruanos cuentan con seguros -en este caso, señala el SIS (Seguro Integral de Salud) - y asume que los migrantes venezolanos lo consiguen de manera inmediata. Todo ello tiene relación con la estrategia de victimización, también propuesta por Van Dijk (2003). Esta última estrategia está vinculada con la polarización, ya que ambas pretenden organizar el discurso en torno a un grupo polarizado: nosotros-ellos. Por otro lado, luego de señalar el aspecto social y sanitario - tomando en cuenta el contexto de pandemia-, el usuario también hace hincapié en el ámbito económico y resalta que, debido a la situación de crisis económica, el país no puede mantener a los migrantes. Más adelante, observamos la estrategia de criminalización cuando el usuario describe a los migrantes venezolanos como delincuentes y lo recalca colocando la nacionalidad entre paréntesis. Esta estrategia pretende describir negativamente a los migrantes y está enlazada con la estrategia de presentación negativa del otro (Van Dijk, 2003). Por último, el usuario utiliza el adjetivo mermelera para describir y presentar negativamente a la prensa, pues considera que los medios no comunican o informan correctamente respecto a lo que el usuario considera como invasión venezolana; es decir, considera al migrante venezolano como invasor.

A continuación, observemos el siguiente tuit, que expresa el rechazo hacia la ayuda social para los migrantes venezolanos: 
(2) No tenemos ni para nosotros mismos!, estamos a las justas y encima tenemos que lidiar y convivir con gente de otro lado que lo unico que hace es empeorar nuestra situacion, y ni hablar de la inseguridad que se tiene con ellos (SunSmile, 2020).

En (2), el usuario elabora un discurso polarizado más explícito al utilizar el pronombre nosotros y los verbos estamos y tenemos para referirse a los peruanos; en cambio, para los migrantes venezolanos utiliza el pronombre ellos. Además, pretende enfatizar su desaprobación utilizando el signo de exclamación al momento de cerrar la siguiente frase: «No tenemos ni para nosotros mismos!». También es importante señalar la denominación que otorga a los migrantes venezolanos: gente de otro lado. Para intensificar su idea, el usuario utiliza en su discurso la estrategia de presentación negativa del otro (Van Dijk, 2003) cuando señala que «lo unico que hace es empeorar nuestra situacion» (SunSmile, 2020). Por último -al igual que en el tuit anterior-, el usuario utiliza la estrategia de criminalización cuando pretende dar a entender que la inseguridad ciudadana está en aumento debido a la presencia de los migrantes venezolanos.

(3) «Los venezolanos ya estan cobrando su bono con los robos diarios que hacen» (Diego, 2020).

En (3), el usuario relaciona la entrega de bonos -subsidios que se brindó a la po- blación vulnerable del Perú y que fueron una de las principales medidas aplicadas por el Estado («Habrá bono de S/ 760 para trabajadores en suspensión perfecta», 2020) - y la imagen criminalizada de los migrantes venezolanos para justificar el rechazo a una posible medida de ayuda social para los migrantes. Esta justificación se observa cuando el usuario señala que «ya estan cobrando su bono con los robos diarios que hacen» (Diego, 2020). Esta tendencia de criminalización continúa en el siguiente tuit:

(4) «No, el gobierno no puede ayudar a personas que han venido a delinquir» (Avilez, 2020).

En (4), la usuaria utiliza el verbo delinquir para presentar una imagen criminalizada del migrante venezolano. De esta manera, observamos que se han utilizado verbos, nombres y adjetivos relacionados con la delincuencia y la inseguridad para representar a los migrantes. La imagen criminal del migrante está basada en estereotipos que se hacen más evidentes en las redes sociales; en consecuencia, los usuarios en contra de la ayuda social para los migrantes venezolanos encuentran en esta imagen estigmatizada una justificación para rechazarlos. Además, es importante señalar que esta percepción no solo se evidencia en el Perú, sino también en otros países de Latinoamérica (Altamirano y Torres-Toukoumidis, 2021), y esto lleva a que se plasmen relaciones de desigualdad y de poder que están basadas en 
los estereotipos previamente señalados (Ramírez Lasso, 2018).

(5) «Buenas tardes no merecen que los ayude dígame usted no siguen trayendo más desgracia al Perú[.]

Lo que debe las Autoridades es Deportarlos ah su Patria de dónde nunca debieron de Salir» (Giorgio.banquero@hot, 2020).

En (5), además del uso de la estrategia de presentación negativa del otro - «Siguen trayendo más desgracia al Perú» (Giorgio. banquero@hot, 2020)-, observamos el uso de la estrategia de imposición de norma. A través de las expresiones de norma - «No merecen que los ayude» $\mathrm{y}$ «Lo que debe las Autoridades es» (Giorgio.banquero@hot, 2020)-, el usuario considera que se encuentra en la posición de decidir por los otros, desde su perspectiva, y establecerse como autoridad. Esta estrategia se relaciona con lo señalado por Gallardo Paúls y Enguix Oliver (2016), ya que los autores consideran que los usuarios, al percibirse consultados, establecen comentarios sin filtros. Usualmente, los discursos que se generan sin filtro están relacionados con los discursos de rechazo; de esta manera, se refuerzan los estereotipos y discursos de odio en las redes sociales (Altamirano y Torres-Toukoumidis, 2021). Por último, el rechazo es más explicito y se agudiza cuando el usuario señala que deben «deportarlos ah su Patria de dónde nunca debieron de Salir»
(6) «No hay ayuda social para los peruanos y tratar de implementar para extranjeros primero cumplan con los peruanos y de hay ver por los extranjeros» (Linares, 2020).

En (6), observamos nuevamente la estrategia de victimización. El usuario señala que no hay ayuda social para los peruanos, lo cual evidencia indignación y rechazo hacia un grupo claramente diferenciado, a quienes denomina como extranjeros -venezolanos-; es decir, no hace explícita la nacionalidad. Más adelante, el usuario utiliza la estrategia principal que observamos en (5): imposición de norma. El usuario considera que el Estado primero debe cumplir con el apoyo para los peruanos y luego tomar en cuenta a los extranjeros con la siguiente expresión de norma: «Primero cumplan con los peruanos y de hay ver por los extranjeros» (Linares, 2020). Por último, destacamos en este tuit que el usuario no ha enfatizado de manera negativa sobre alguna característica física o psicológica del migrante y tampoco ha utilizado algún tipo de comentario o adjetivo calificativo de manera despectiva contra los migrantes. Interpretamos este tuit como una crítica mesurada en comparación con los anteriores.

En el siguiente tuit (7), observamos una respuesta de un usuario peruano que interactuó con el tuit de un usuario venezolano:

(7) No deberías abusar de los buenos peruanos, recuerda que de los malos 
peruanos y de los que comentan en contra TAMBIÉN VIVES. Estas en su casa, aprende a respetarlos. Y si llegamos a una crisis ustedes fueron parte del PROBLEMA (Itaca, 2020).

En (7), el usuario peruano interactúa - responde un tuit- con un usuario venezolano que agradecía a los peruanos de buen corazón que comentan cosas positivas y decía que, si bien hay malos venezolanos, también hay buenos, los cuales son mayoría. Ante ello, el usuario polariza en grupos a los peruanos basado en dos principales adjetivos: buenos peruanos y malos peruanos. En esta línea, el usuario peruano le sugiere al usuario venezolano que no debe abusar de los buenos peruanos y tampoco debe comentar algo en contra de los malos peruanos, ya que considera que los migrantes venezolanos viven a costa de ambos grupos, sean buenos o malos. De esta manera, observamos que busca justificar los actos xenófobos que puedan experimentar los migrantes. Además, otro aspecto por resaltar es el uso de las letras mayúsculas por parte del usuario para intensificar el mensaje que pretende resaltar; por ejemplo, destaca la construcción TAMBIÉN VIVES y el sustantivo PROBLEMA. Por último, evidencia una estrategia denominada evasión (Aedo y Farías, 2009). Esta estrategia permite que el usuario, en su discurso, evada la responsabilidad -la crisis, en este caso - y se la otorgue a terceros - «Y si llegamos a una crisis ustedes fueron parte del PROBLEMA» (Itaca, 2020)-.
(8) COMO TIENEN PLATA PARA CHUPAR TODOS LOS FINES DE SEMANA... ROPA DE MARCA...ETC YA TIENEN MOTOS AUTOS MOTOTAXIS ...??? MAS BENEFICIOS DEL GOBIERNO... EXISTEN BARES EXCLUSIVOS PARA ELLOS Y LOS FINES DE SEMANA...ESTAN BIEN PERCHADOS CHUPANDO... NO TRABAJAN LUNES..??? TONCES (JRigo Quispe H, 2020).

En (8), de igual manera que en el tuit anterior, destacamos el uso de las letras mayúsculas -en este caso, durante todo el tuit- por parte del usuario, con la finalidad de intensificar su mensaje. Incluso, podemos considerar que expresa, de manera exaltada y manifiesta, un rotundo rechazo ante la consulta de apoyo social a favor de los migrantes venezolanos. Por otro lado, en el tuit, el usuario utiliza -como observamos en los ejemplos anteriores- la estrategia de presentación negativa. En primer lugar, utiliza en dos ocasiones el verbo chupar (beber alcohol); es decir, relaciona a los venezolanos con las bebidas alcohólicas y las fiestas, lo cual explicita aún más al indicar que tienen bares exclusivos donde pueden beber alcohol libremente - «EXISTEN BARES EXCLUSIVOS PARA ELLOS» (JRigo Quispe H, 2020)-. Luego, utiliza ropa de marca, motos, autos y mototaxis para dar a entender que los migrantes venezolanos cuentan con el dinero suficiente para obtener objetos caros y vestir ropa de marca -«ESTÁN BIEN PERCHADOS» (JRigo Quispe H, 2020)-. 
(9) Hasta ahora muchos Peruanos esperamos el bono y se van a preocupar por esta lacra gente de mierda q solo vino a robar asesinar dar problemas y acabar con la poca seguridad y tranquilidad que teníamos Peruanos q los ayude Maduro (Maluco, 2020).

En el caso de (9), destacamos que el usuario ha utilizado el recurso del insulto. Bonet-Martí (2020) considera que este recurso está vinculado con la amenaza y es la principal modalidad de los discursos de odio para representar el racismo y la xenofobia; además, este tipo de recursos son comunes en los discursos de rechazo en las redes sociales. Observamos que el usuario evidencia el uso de este recurso a través del adjetivo lacra y la construcción gente de mierda. Asimismo, no se puede dejar de lado la tendencia al uso de estrategias constantemente utilizadas: la polarización a través de pronombres, la presentación negativa del otro y la criminalización.

Finalmente, observemos el último tuit de esta primera sección:

(10) Somos concientes de la nesecidad de los venezolanos, pero hoy en dia, nuestro pais atravieza por una algida crisis economica, por lo tanto se tiene que priorizar a nuestros compatriotas, los extrajeros tendran que ver si continuan aca o regresan a su pais. No hay para ellos (Pérez, 2020).
En (10), destacamos el uso de la estrategia de concesión aparente (Aedo y Farías, 2009). Esta estrategia usualmente se evidencia con el uso de un marcador de contraste - pero, sin embargo, etcétera-. El usuario, aparentemente, cede ante la necesidad de los venezolanos; sin embargo, continúa la elaboración de su tuit con el uso de un conector de contraste - pero-. Luego, para justificar su oposición a la primera idea, destaca la situación crítica de la economía del Perú con el adjetivo álgido. Por esta razón, polariza a los peruanos en un grupo - nuestros compatriotas - y en otro a los migrantes venezolanos -extranjeros-. Por último, el usuario finaliza el tuit aplicando la estrategia de imposición de norma y señala que los migrantes deben decidir entre esperar por la ayuda o irse del país, ya que «no hay para ellos» (Pérez, 2020).

\section{«En momentos de pandemia no hay nacionalidades»}

En esta segunda sección, se analizan los tuits con respuestas y comentarios positivos y a favor de la ayuda social para los migrantes venezolanos. El discurso de en momentos de pandemia no hay nacionalidades es una constante en esta sección. Por ejemplo, observemos el siguiente tuit:

(11) En momentos de pandemia no hay nacionalidades, se debe apoyar a todos por igual ,dando las mismas oportunidades en muchas partes del mundo hay peruanos y venezolanos 
trabajando , dejando a su país orgulloso, no todos son malos hay más buenos que malos ... (Valles, 2020).

En (11), el usuario señala que las nacionalidades se deben dejar de lado cuando se trata de asistir a una comunidad vulnerable en contexto de pandemia - «En momentos de pandemia no hay nacionalidades» (Valles, 2020)-; es decir, considera que se debe brindar el apoyo indistintamente de la nacionalidad, invisibilizar las nacionalidades. Además, el usuario agrega que se deben brindar las mismas oportunidades; de esta manera, evidencia aún más aprobación a una posible ayuda social en beneficio de los migrantes venezolanos. Más adelante, utiliza la estrategia de la empatía en el discurso cuando señala que, en otros países, también hay peruanos establecidos y que muchos, en algunos casos, deben pasar por una situación similar. Finalmente, polariza a los migrantes venezolanos en dos grupos, que están basados en los adjetivos calificativos buenos y malos - «No todos son malos hay más buenos que malos» (Valles, 2020)- e indica que los buenos venezolanos son mayoría.

(12) «Yo creo que si,ellos son nuestros hermanos,y son seres humanos con las mismas necesidades igual que nostros. Recordemos que tambien hay peruanos en otros paises» (Medina Arroyo, 2020).

En (12), observamos que la estrategia de la empatía en el discurso continúa cuan- do el usuario señala que debemos recordar que «tambien hay peruanos en otros paises» (Medina Arroyo, 2020); con ello, se refiere a los peruanos en el extranjero que, en muchos casos, también necesitan apoyo por parte de los Gobiernos de los países donde se encuentran. Otro aspecto por destacar es el uso de la hermandad entre países en el discurso. El usuario evidencia esta estrategia al momento de señalar que ellos son nuestros hermanos; de esta manera, expresa el apoyo mutuo que considera que se deben tener peruanos y venezolanos. Finalmente, recalca que, de igual manera que los peruanos, los migrantes venezolanos son seres humanos y que, en consecuencia, tienen las mismas necesidades y carencias: «Son seres humanos con las mismas necesidades igual que nostros» (Medina Arroyo, 2020). La estrategia de humanizar al migrante venezolano continúa en el siguiente tuit:

(13) En medio de una PANDEMIA no debe de existir nacionalidades ya que el virus no es ajeno a nadie. $Y$ en el sistema económico también dificulta a todas las personas de un país así sean extranjeros. Debemos apoyarnos entre humanos ya que todos somos vulnerables, el gob apoye a todos (Navarro, 2020).

En el caso de (13), también observamos la estrategia de humanizar al migrante venezolano en el discurso - «Debemos apoyarnos entre humanos ya que todos somos vulnerables» (Navarro, 2020)-. 
Además, en este tuit, destacamos que el usuario utiliza las letras mayúsculas para intensificar la palabra pandemia, pero -a diferencia de los tuits de la primera sección- esta intensificación no tiene una carga negativa. El usuario utiliza esta estrategia para resaltar la grave situación en la que se encuentran el Perú y el resto de países en el mundo. Más adelante, resalta la situación económica del país para asumir que ahora los migrantes venezolanos en el Perú son parte del territorio; en consecuencia, no se evidencia la polarización. Finalmente, el usuario considera que los migrantes no son parte del problema y que deben apoyarse mutuamente - esto también se ejemplifica con el enunciado «Debemos apoyarnos entre humanos ya que todos somos vulnerables» (Navarro, 2020)-.

(14) «Si se debe crear programas para ayuda, la ayuda no tiene nacionalidad, no deberia ser excluyente» (Dorador, 2020).

En (14), el usuario expresa su aprobación ante una posible ayuda social por parte del Estado a favor de los migrantes venezolanos a través del uso de las estrategias de la hermandad y la invisibilización de las nacionalidades. Esto se evidencia cuando el usuario señala que la diferencia de nacionalidades en contexto de pandemia no debe ser un obstáculo para generar programas de ayuda social para todos; es decir, apunta a no diferenciar entre peruanos y venezolanos para recibir ayuda por parte del Gobierno $-\ll \mathrm{La}$ ayuda no tiene nacionalidad» (Dorador, 2020)-. Por esta razón, considera que la ayuda social no debe ser excluyente; de esta manera -al igual que en los tuits anteriores-, el usuario construye un discurso inclusivo.

(15) «Obvio q sí. Son personas mayormente sin recursos y muchos de ellos aportan a la economía del país. Son seres humanos. Y sería una forma de empadronarlos y saber a q se dedican tmb» (JB, 2020).

Finalmente, en (15), el usuario utiliza la estrategia de humanizar al migrante cuando resalta que un gran grupo de migrantes venezolanos en el Perú atraviesa por una crisis económica que les impide cumplir con los criterios básicos de habitabilidad humana - «Son personas mayormente sin recursos» (JB, 2020)-. Además, presenta positivamente a los venezolanos - «Muchos de ellos aportan a la economía del país» (JB, 2020)-. Esto se diferencia con los tuits de la primera sección, en los que la presentación era negativa. Asimismo, observamos que el usuario elabora un comentario a favor de los venezolanos en un tema polémico como la economía, el cual era la principal inquietud en los comentarios en contra de la inclusión. Por último, contribuye positivamente al indicar que otorgarles algún tipo de ayuda social podría favorecer el empadronamiento para tener un mayor control y conocimiento respecto a los empleos de una comunidad que cada vez se expande más en el país. 


\section{Conclusiones}

En conclusión, los usuarios peruanos, ante la encuesta en línea elaborada por la cuenta en la red social Twitter del canal de televisión de noticias ATV+ Noticias - «iCree usted que el gobierno debe implementar programas de ayuda social para los venezolanos que atraviesan la crisis de la pandemia?» (2020)-, evidencian y representan una posición marcadamente polarizada: a favor y en contra. Sin embargo, se hace explícita una mayor inclinación hacia los comentarios negativos. En ambas posturas, los usuarios utilizan estrategias y recursos en sus tuits para fortalecer y sustentar su mensaje.

En el caso de los tuits en contra de la implementación de programas de ayuda social para los migrantes venezolanos, los usuarios peruanos de esta red social emplean las siguientes estrategias: la polarización, la victimización, la criminalización, la presentación negativa del otro, la imposición de norma, el uso de las letras mayúsculas para intensificar el mensaje, la evasión, el recurso del insulto y la concesión aparente. Estas estrategias y recursos buscan establecer dos grupos marcadamente distintos entre peruanos y venezolanos. En esta línea, se describe a estos últimos de manera despectiva -invasores, ladrones, gente de otro lado, etcétera-y se los culpa por la crisis que atraviesa el país. Todo ello se manifiesta con la finalidad de justificar el rechazo de que el Estado les otorgue una posible ayuda social.
Por último, en el caso de los tuits a favor de la implementación de programas de ayuda social para los migrantes venezolanos, los usuarios peruanos emplean las siguientes estrategias: la empatía en el discurso, el recurso de hermandad entre países, invisibilizar las nacionalidades, el uso de las letras mayúsculas para intensificar el mensaje, humanizar al migrante, la presentación positiva y la inclusión. Estas estrategias y recursos buscan no polarizar entre peruanos y venezolanos, ya que los usuarios peruanos a favor de la iniciativa perciben a los migrantes venezolanos como hermanos -ellos son nuestros hermanos-, los describen positivamente - muchos de ellos aportan a la economía del país- y empatizan con ellos. 


\section{REFERENCIAS}

ACNUR. (2019, 22 de febrero). Los flujos de venezolanos continúan constantes, alcanzando ahora la cifra de 3,4 millones [Comunicado de prensa]. https:// bit.ly/2PuQvgb

Aedo, S. y Farías, M. (2009). Etnofaulismos, coprolalia, representaciones y estrategias discriminatorias: el caso del discurso chileno antiperuano. Discurso \& Sociedad, 3(3), 372-396. http://www.dissoc.org/ediciones/ vo3no3/DS3(3)Aedo\&Farias.pdf

Altamirano, G. F. y Torres-Toukoumidis, Á. (2021). Análisis del discurso xenófobo hacia la migración venezolana en los comentarios de las publicaciones de Facebook pertenecientes a los diarios locales: El Mercurio y El Tiempo. GIGAPP Estudios Working Papers, 8(211), 310-325. http://www.gigapp. org/ewp/index.php/GIGAPP-EWP/ article/view/259

ATV+ Noticias [@atvmasnoticias]. (2020, 24 de septiembre). Participa en la pregunta del día de \#EnContacto con @VertizPamela ¿Cree usted que el gobierno debe implementar programas de ayuda [Tuit]. Twitter. https:// twitter.com/atvmasnoticias/status/1309168686259941376

Avilez, A. [@AliciaAvilez8]. (2020, 24 de septiembre). No, el gobierno no puede ayudar a personas que han venido a delinquir [Tuit]. Twitter. https:// twitter.com/AliciaAvilez8/status/1309181283810054144
Banco Mundial. (2020, 8 de junio). La COVID-19 (coronavirus) hunde a la economía mundial en la peor recesión desde la Segunda Guerra Mundial [Comunicado de prensa]. https:// www.bancomundial.org/es/news/ press-release/2020/06/08/covid19-to-plunge-global-economy-intoworst-recession-since-world-war-ii

Barrutia Barreto, I., Sánchez Sánchez, R. M. y Silva Marchan, H. A. (2021). Consecuencias económicas y sociales de la inamovilidad humana bajo Covid 19 caso de estudio Perú. Lecturas de Economía, (94), 285-303. https://doi. org/10.17533/udea.le.n94a344397

Bonet-Martí, J. (2020). Análisis de las estrategias discursivas empleadas en la construcción de discurso antifeminista en redes sociales. Psicoperspectivas. Individuo y Sociedad, 19(3), 52-63. https://dx.doi.org/10.5027/ psicoperspectivas-vol19-issue 3 -full$\underline{\text { text-2040 }}$

Coronavirus en Perú: «Vamos a mantener la calma y confiar en el sistema de salud», dice Martín Vizcarra. (2020, 6 de marzo). El Comercio. https://bit. ly/3nISDgO

DataReportal. (2021, julio). Global social media stats. https://datareportal.com/ social-media-users

Decreto Supremo N 046-2020-PCM [Presidencia del Consejo de Ministros]. (2020, 18 de marzo). Decreto Supremo que precisa el Decreto Supremo No ${ }^{\circ} 4^{-}$ 2020-PCM, que declara el Estado de Emergencia Nacional, por las graves circunstancias que afectan la vida de la Nación a consecuencia del brote del COVID 19. Diario Oficial El Peruano. https://bit.ly/2QjVggP 
Defensoría del Pueblo. (2020). Personas venezolanas en el Perú. Análisis de la situación antes y durante la crisis sanitaria generada por el covid-19. https://bit.ly/3f3JD3n

Diego [@Diego34090855]. (2020, 24 de septiembre). Los venezolanos ya estan cobrando su bono con los robos diarios que hacen [Tuit]. Twitter. https://twitter.com/Dieg034090855/ status/1309178639200792576

Dorador, R. [@ricardodorador]. (2020, 24 de septiembre). Si se debe crear programas para ayuda, la ayuda no tiene nacionalidad, no deberia ser excluyente [Tuit]. Twitter. https:// twitter.com/ricardodorador/status/1309258256989278212

Gallardo Paúls, B. y Enguix Oliver, S. (2016). Pseudopolítica: el discurso político en las redes sociales. Universitat de València. https://roderic.uv.es/handle/10550/66756

Giorgio.banquero@hot [@BanqueroGiorgio]. (2020, 24 de septiembre). Buenas tardes no merecen que los ayude dígame usted no siguen trayendo más desgracia al Perú Lo que debe las [Tuit]. Twitter. https:// twitter.com/BanqueroGiorgio/status/1309227418025168920

Gobierno del Perú. (2021). Coronavirus: apoyos económicos del Estado por el aislamiento social obligatorio. https://www.gob.pe/8895-coronavirus-apoyos-economicos-del-estado-por-el-aislamiento-social-obliga$\underline{\text { torio }}$
Gómez Walteros, J. A. (2010). La migración internacional: teorías y enfoques, una mirada actual. Semestre Económico, 13(26), 81-99. http://www.scielo.org. co/scielo.php?script=sci arttext\&pi$\mathrm{d}=$ S0120-63462010000100005\&ln$\mathrm{g}=\mathrm{en} \& \operatorname{tlng}=\mathrm{es}$

Gonzales-Castillo, J. R., Varona-Castillo, L., Dominguez-Morante, M. G. y Ocaña-Gutierrez, V. R. (2020). Pandemia de la COVID-19 y las Políticas de Salud Pública en el Perú: marzo-mayo 2020. Revista de Salud Pública, 22(2), 1-9. https://doi.org/10.15446/rsap. v22n2.87373

Habrá bono de S/ 760 para trabajadores en suspensión perfecta que ganen hasta S/ 2,400. (2020, 24 de junio). Gestión. https://bit.ly/zetXjUR

Hernández Sampieri, R. y Mendoza, C. P. (2018). Metodología de la investigación. Las rutas cuantitativa, cualitativa y mixta. McGraw-Hill Interamericana.

Huaman Fernández, J. R. (2021). Impacto económico y social de la covid-19 en el Perú. Revista de Ciencia e Investigación en Defensa-CAEN, 2(1), 31-42. http://recide.caen.edu.pe/index. php/Recide/article/view/49

Hütt Herrera, H. (2012). Las redes sociales: una nueva herramienta de difusión. Revista Reflexiones, 91(2), 121-128. https://revistas.ucr.ac.cr/index.php/ reflexiones/article/view/1513 
Instituto Nacional de Estadística e Informática. (2019). Condiciones de vida de la población venezolana que reside en Perú. Resultados de la «Encuesta dirigida a la población venezolana que reside en el país» ENPOVE 2018. https://www.inei.gob.pe/media/MenuRecursivo/publicaciones digitales/ Est/Lib1666/

Itaca [@Itaca77227690]. (2020, 24 de septiembre). No deberías abusar de los buenos peruanos, recuerda que de los malos peruanos y de los que comentan en contra [Tuit]. Twitter. https://twitter.com/Itaca77227690/ status/1309281703953866757

JB [@Txalooo7]. (2020,25 de septiembre). Obvio $q$ sí. Son personas mayormente sin recursos y muchos de ellos aportan a la economía del país. Son seres [Tuit]. Twitter. https://twitter.com/Txalooo /status/1309504577365041154

JRigo Quispe H [@Yeshuahqh]. (2020, 24 de septiembre). COMO TIENEN PLATA PARA CHUPAR TODOS LOS FINES DE SEMANA...ROPA DE MARCA... ETC YA TIENEN MOTOS AUTOS MOTOTAXIS ...??? MAS [Tuit]. Twitter. https://twitter.com/Yeshuahqh/sta$\underline{\text { tus} / 1309170028848312321}$

Koechlin, J., Vega, E. y Solórzano, X. (2018). Migración venezolana al Perú: proyectos migratorios y respuesta del Estado. En J. Koechlin y J. Eguren (Eds.), El éxodo venezolano: entre el exilio y la emigración (pp. 47-96).
Linares, C. [@CarlosL97360569]. (2020, 24 de septiembre). No hay ayuda social para los peruanos y tratar de implementar para extranjeros primero cumplan con los peruanos y de [Tuit]. Twitter. https:// twitter.com/CarlosL97360569/status/1309177532936224773

Lovón Cueva, M. A. y Chegne Cortez, D. A. (2021). Repercusión del aislamiento social por COVID-19 en la salud mental en la población de Perú: síntomas en el discurso del ciberespacio. Discurso \& Sociedad, 15(1), 215-243. http://www.dissoc.org/ediciones/ v15n01/DS15(1)Lovon\&Chegne.html

Maluco [@Bellobe70610488]. (2020, 24 de septiembre). Hasta ahora muchos Peruanos esperamos el bono y se van a preocupar por esta lacra gente de mierda q solo [Tuit]. Twitter. https:// twitter.com/Bellobe70610488/status/1309172787676958723

Medina Arroyo, P. [@PercyMedinaArr1]. (2020, 24 de septiembre). Yo creo que si,ellos son nuestros hermanos,y son seres humanos con las mismas necesidades igual que nostros. Recordemos [Tuit]. Twitter. https:// twitter.com/PercyMedinaArr1/status/1309179119150694401

Meersohn, C. (2005). Introducción a Teun Van Dijk: análisis de discurso. Cinta de Moebio. Revista de Epistemología de Ciencias Sociales, 24, 288-302. https://www.moebio.uchile.cl/24/ meersohn.html 
Navarro, L. [@Luoo_navarro]. (2020,24 de septiembre). En medio de una PANDEMIA no debe de existir nacionalidades ya que el virus no es ajeno a nadie. $Y$ [Tuit]. Twitter. https:// twitter.com/Luoo navarro/status/1309176585975668736

Superintendencia Nacional de Migraciones. (2019). Memoria 2018-2019. Transformación digital y modernización del Estado. https://cdn. www.gob.pe/uploads/document/ file/1260560/MEMORIA MIGRACIONES 2018 2019.pdf

Ministra Donayre: Programas sociales se han fortalecido durante la pandemia. (2020, 25 de setiembre). El Peruano. https://elperuano.pe/noticia/104342-ministra-donayre-programas-sociales-se-han-fortalecido-durante-la-pandemia

Observatorio Nacional de las Telecomunicaciones y de la SI. (2011, diciembre). Las Redes Sociales en Internet. https:// www.ontsi.red.es/sites/ontsi/files/redes sociales-documento o.pdf

Pérez, K. [@perez_sovero]. (2020, 24 de septiembre). Somos concientes de la nesecidad de los venezolanos, pero hoy en dia, nuestro pais atravieza por una algida crisis economica [Tuit]. Twitter. https://twitter.com/perez sovero/status/1309501495931535361

Pérez Abreu, M. R., Gómez Tejada, J. J. y Dieguez Guach, R. A. (2020). Características clínico-epidemiológicas de la COVID-19. Revista Habanera de Ciencias Médicas, 19(2), Artículo e3254. http://www.revhabanera.sld.cu/index.php/rhab/article/ view/3254/2505
Pérez Matar, R. y Paredes Esponda, E. (2016). Red Social Twitter. Fortalezas y Debilidades. Infodir, (23), 77-86. http:// www.revinfodir.sld.cu/index.php/ infodir/article/view/204

Portillo Fernández, J. (2016). Planos de realidad, identidad virtual y discurso en las redes sociales. Logos. Revista de Lingüística, Filosofía y Literatura, 26(1), 51-63. https://dx.doi. org/10.15443/RL2604

Ramírez Lasso, L. M. (2018). Representaciones discursivas de las migrantes venezolanas en medios digitales. Revista Latinoamericana de Estudios del Discurso, 18(2), 42-58. http://dx.doi. org/10.35956/v.18.n2.2018.p.42-58

Rommel [@rommelred11]. (2020, 24 de septiembre). Primero están lo peruanos, hay muchos compatriotas que no tienen ni SIS y a los venezolanos ni bien llegan les [Tuit]. Twitter. https://twitter.com/rommelred11/status/1309180655583010818

SunSmile [@SunSmil79252354]. (2020, 24 de septiembre). No tenemos ni para nosotros mismos!, estamos a las justas y encima tenemos que lidiar y convivir con gente de [Tuit]. Twitter. https://twitter.com/SunSmil79252354/ status/1309178661535444994

Suurmond, J. M. (2005). Our talk and walk: Discourse analysis and conflict studies. Netherlands Institute of International Relations, Working paper 35. Netherlands Institute of International Relations. https://www. clingendael.org/sites/default/files/ pdfs/20051000 cru working paper $35 . \mathrm{pdf}$ 
Urra, E., Muñoz, A. y Peña, J. (2013). El análisis del discurso como perspectiva metodológica para investigadores de salud. Enfermería Universitaria, 10(2), 50-57. http://www.scielo.org. $\mathrm{mx} /$ scielo.php?script $=$ sci arttex$\underline{\mathrm{t} \& \mathrm{pid}=\mathrm{S} 1665-70632013000200004}$

Valles, M. [@ManuelV98799839]. (2020, 24 de septiembre). En momentos de pandemia no hay nacionalidades, se debe apoyar a todos por igual ,dando las mismas oportunidades en muchas [Tuit]. Twitter. https:// twitter.com/ManuelV98799839/status/1309194332193390592

Van Dijk, T. A. (2003). Ideología y discurso. Una introducción multidisciplinaria. Ariel.

Van Dijk, T. A. (2016). Análisis Crítico del Discurso (Trads. C. Büchner y C. Cárdenas). Revista Austral de Ciencias Sociales, (30), 203-222. https:// doi.org/10.4206/rev.austral.cienc. soc.2016.n30-10 (Trabajo original publicado en 2015) 